\title{
COMPARISON BETWEEN H.264/AVC AND MOTION JPEG2000 FOR SUPER-HIGH DEFINITION VIDEO CODING
}

\author{
Chenwei Deng, Weisi Lin, Bu-sung Lee, Chiew Tong Lau and Manoranjan Paul
}

School of Computer Engineering, Nanyang Technological University, Singapore, 639798

\begin{abstract}
H.264/AVC FRExt (Fidelity Range Extensions) and Motion JPEG2000 are the latest inter-frame and intra-frame video coding standards, respectively. It is well known that an interframe method achieves higher coding efficiency compared with an intra-frame one, and the Motion JPEG2000 has been selected for digital cinema compression. In this paper, we attempt to compare these two different schemes with theoretical and experimental analysis for super-HD (high definition) visual signals. One additional contribution of the paper is that the impact of block partition, motion search range and skipped block size for inter-frame coding is discussed. Based on the analysis, we extend the standard H.264/AVC FRExt by using larger block size and search range. The experimental results show that this extension leads to higher coding efficiency and makes the H.264/AVC FRExt more suitable for super-HD video coding.
\end{abstract}

Index Terms - H.264/AVC FRExt, Motion JPEG2000, Video coding, Digital cinema, Super-high resolution

\section{INTRODUCTION}

With the advance of digital signal processing technology, digital cinema and high definition TV (HDTV) have been revolutionizing entertainment industry due to the superior video quality they can offer. Digital cinema is with super-HD video (e.g., a $4096 \times 2160$ video frame contains more than eight megapixels). The Digital Cinema Initiatives (DCI) has established a specification [1], in which the Motion JPEG2000 has been selected as the compression standard for digital cinema.

The Motion JPEG2000 is derived from Part 1 of ISO/ IEC 15444-1 standard, and it exploits spatial redundancy to achieve data compression (i.e., intra-frame coding). This is in contrast with the H.264/AVC FRExt, which exploits both spatial and temporal redundancy (i.e., inter-frame coding). It is commonly believed that inter-frame coding is more efficient than intra-frame coding in terms of rate-distortion (R-D) performance. Does the H.264/AVC FRExt have any advantage in the case of super-HD video coding (e.g., digital cinema sequences)?

This work was supported by MoE AcRF Tire 2, Singapore, Grant Number: T208B 1218
Smith et al. [2] tested the coding efficiency differences between inter-frame and intra-frame architectures for high quality, HD sequences. Shi et al. [3] compared the objective and subjective performance of JPEG2000, H.264 intra and the full H.264. Baruffa et al. [4] presented an assessment of the JPEG2000-based motion compensated temporal filtering and the H.264/AVC FRExt. These experiments claimed that the Motion JPEG2000 is more suitable for digital cinema compression; however, such conclusion was mainly based on experimental results and without any theoretical analysis.

In this paper, we attempt to understand the related issues and give more insight, from both theoretical and experimental points of view. The performance of the H.264/ AVC FRExt and Motion JPEG2000 is to be first compared, the comparison results are then analyzed, and the impact of block partition, search range and skipped block size for motion compensated inter-frame coding are discussed. Based upon the results of the said analysis, we extend the H.264/ AVC FRExt for better super-HD video coding.

The rest of the paper is organized as follows: Section 2 compares the R-D performance between the H.264/AVC FRExt and the Motion JPEG2000 using multiple super-HD test sequences; Section 3 gives the theoretical analysis on the impact of different related inter-frame coding parameters toward HD content, and provides the ground for extending the standard H.264/AVC FRExt for super-HD sequences; Section 4 demonstrates experimental results of the extended H.264/AVC FRExt, while Section 5 concludes this paper.

\section{COMPARISON ON R-D PERFORMANCE}

In this section, we are to test the coding efficiency of the H.264/AVC FRExt and Motion JPEG2000. Three 4096×2160 test sequences "Pedestrian area", "Crowd Run" and "Park joy" (as shown in Fig.1) are used for comparison; the first video clip is with still camera and low motion and the second one is with still camera but high motion, while the last one is with moving camera and high motion. Each frame has 12 bits per color and 4:4:4 color sampling.

JM 16.2 [5] is used for H.264/AVC FRExt compression. In contrast with [3] where H.264 High Profile (HP) at Level 4.1 was used to encode $4096 \times 2160$ test sequences, in our experiments, we used H.264/AVC High Profile at Level 5.1 


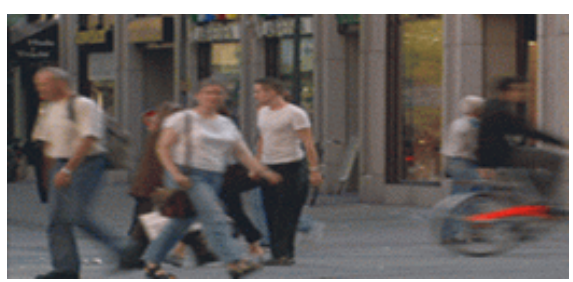

(a) Pedestrian area

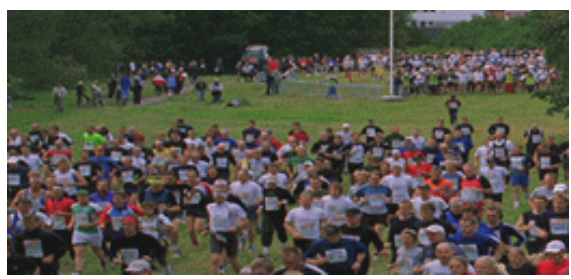

(b) Crowd Run

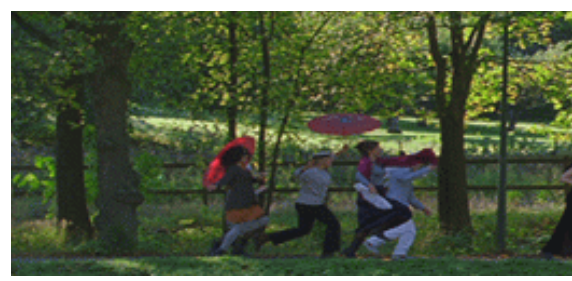

(c) Park joy

Fig. 1: Three $4096 \times 2160$ super-HD (high definition) test sequences.

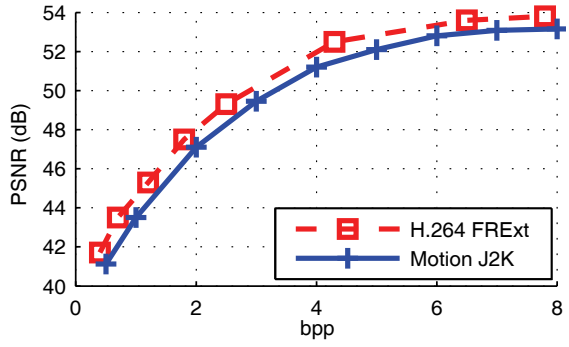

(a) Pedestrian area

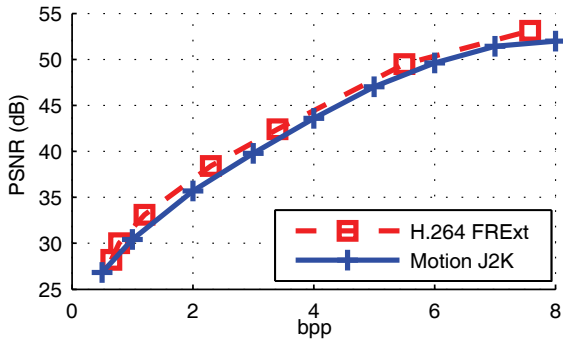

(b) Crowd Run

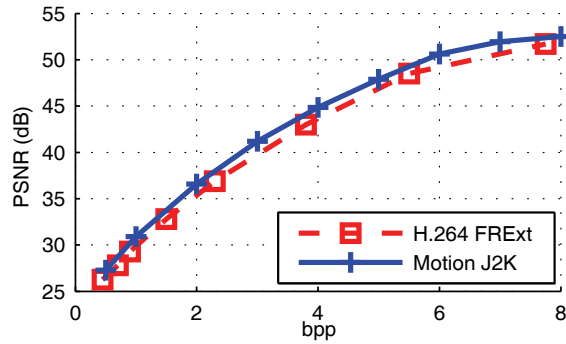

(c) Park joy

Fig. 2: The rate-distortion (R-D) performance (luma component) comparison between standard H.264/AVC FRExt and Motion JPEG2000.

for the simulation (because the maximum resolution for the Level 4.1 is only $2048 \times 1024$ [6]). The size of GOP (Group of Picture) was 16 and with "IBBPBBP..." structure. For P-type and B-type frames, we used one reference frame for inter motion search. Other parameters remained as default values in JM 16.2. Kakadu 6.3.1 [7] was used for the Motion JPEG2000. Five-level Daubechies 9/7 discrete wavelet transform (DWT) was performed.

The test results for the H.264/AVC FRExt and Motion JPEG2000 were presented in Fig.2. We can see that the PSNR advantage of the H.264/AVC FRExt is very small, and for high motion video sequences (e.g., "Park joy"), the Motion JPEG2000 even outperforms the H.264/AVC FRExt.

\section{THEORETICAL ANALYSIS ON CODING PARAMETERS}

As aforementioned, Fig. 2 demonstrates that for super-HD video sequences, the advantage of inter-frame coding is not as significant as in the standard definition (SD) video coding, in comparison with intra-frame coding. We will discuss the reasons for such change from a theoretical viewpoint, and the possibility for the H.264/AVC FRExt to be extended for super-HD video coding to maintain its advantage.

\subsection{Macroblock partition}

In the H.264/AVC FRExt, each frame is partitioned into a sequence of $16 \times 16$ macroblocks. For motion-compensated prediction, each macroblock can be further divided into blocks with seven different sizes - $16 \times 16,16 \times 8,8 \times 16,8 \times 8,8 \times 4$, $4 \times 8,4 \times 4$. The optimal partition can be selected by minimizing the cost function $J(m v, \lambda)$ defined as:

$$
J(m v, \lambda)=\sum_{k} \sum_{i} \sum_{j}\left|C_{i, j}^{k}-R_{i, j}^{k}\right|+\lambda R(m v)
$$

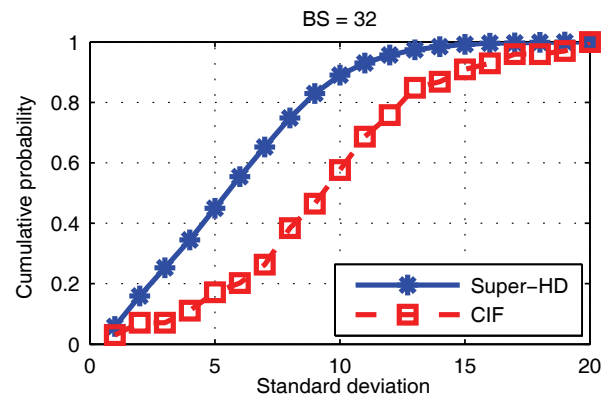

Fig. 3: Cumulative probability of the standard deviation (STD) of $32 \times 32$ blocks. The average frame of "Pedestrian area", "Crowd Run" and "Park joy" was tested.

where $R(m v)$ denotes the rate function with respective to the motion vectors $m v ; C_{i, j}$ and $R_{i, j}$ are the current and reference blocks, respectively; $k$ is the block index; and $\lambda$ is the optimization parameter.

For each block, motion estimation (ME) is performed. It is obvious that those with small block size (BS) (e.g., $8 \times 4$, $4 \times 8,4 \times 4$, etc.) require more bits for motion vectors, but the resultant residuals may contain less energy. In general, a large $\mathrm{BS}$ is suitable for homogeneous region while a small BS may be beneficial for texture.

The partition method above could achieve high coding efficiency for SD sequences. However, this may not be the optimal solution for super-HD (e.g., $4096 \times 2160)$ clips. With a same video content, a super-HD sequence is generally more homogeneous than a lower definition sequence. As shown in Fig. 3, in the super-HD case, over $50 \%$ of the $32 \times 32$ blocks is with the standard deviation (STD) being lower than 6 , while in the CIF case, only $20 \%$ of the $32 \times 32$ blocks meets the same STD criteria. Therefore, for a super-HD sequence, we can allocate fewer bits to the motion vectors by expanding macroblock size and using $32 \times 32,32 \times 16,16 \times 32,16 \times 16$, $16 \times 8,8 \times 16,8 \times 8,8 \times 4,4 \times 8$, and $4 \times 4$ blocks for ME. 

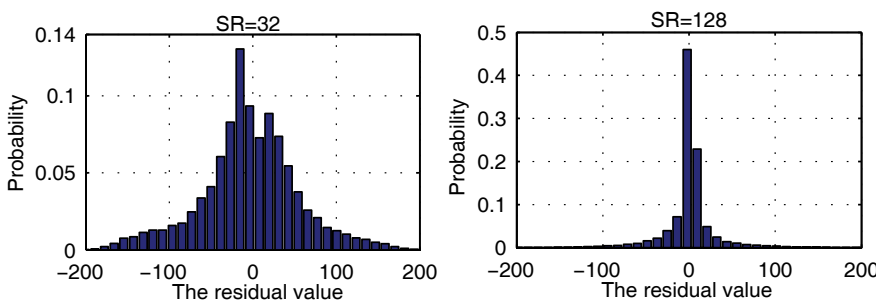

Fig. 4: Normalized distribution of average residuals of "Pedestrian area", "Crowd Run" and "Park joy" using different search range.

\subsection{Motion estimation (ME)}

Block-based ME is one of the bases of the H.264/AVC FRExt. Practically, each $M \times N(M, N=4,8,16)$ block in the current frame is compared with some possible $M \times N$ regions (within a fixed search range) in the reference frames, and the candidate region that minimizes the SAD (sum of absolute differences) or MAD (mean absolute differences) of the residual block is selected for motion compensation.

Due to the fixed search range (SR), the energy of resultant residuals is the local minimal value. For super-HD video sequences, the motion is higher than that of low resolution video with same visual content, and therefore, the SR used for SD sequences cannot capture moving objects in super-HD sequences. In this case, ME efficiency would be significantly affected.

Based on the analysis above, for super-HD video coding, a relatively larger region has to be searched in the reference frames. Fig. 4 explains this, with the notes that by using SR= 128 , the distribution of residuals is more concentrated than that by using $\mathrm{SR}=32$.

Mathematically, residual data can be modeled as a zeromean Laplacian distribution [8]. The probability density function (pdf) is given by

$$
p(r)=\frac{1}{\sqrt{2} \sigma_{r}} \exp \left(-\frac{\sqrt{2}|r|}{\sigma_{r}}\right)
$$

where $\sigma_{r}$ is the STD of the residuals $(r)$ in pixel domain, and $p(r)$ denotes the pdf. Based on Shannon's theorem, the entropy of the residuals can be expressed as

$$
H(r)=-\sum_{-\infty}^{+\infty} p(r) \log _{2}[p(r)]=\frac{\sqrt{2}}{\ln (2)} \sigma_{r}
$$

where $H(r)$ denotes the entropy, and is proportional to $\sigma_{r}$. For a Laplacian distribution, the probability that a value falls within $\left(-3 \sigma_{r}, 3 \sigma_{r}\right)$ is about $99 \%$. From Fig. 4, we can see that $\sigma_{r-S R=128}<\sigma_{r-S R=32}$, and therefore, $H(S R=$ $128)<H(S R=32)$. In this case, a lower QP (quantization parameter) can be used to achieve higher entropy coding performance when the SR is large.

\subsection{Skipped block}

In the H.264/AVC FRExt, if the motion of a block can be effectively predicted from the neighboring blocks, all the transformed coefficients are quantized to zeros, and then the block is flagged as skipped one. The size of P_Skip and B_Skip blocks is $16 \times 16$ and $8 \times 8$, respectively. For a skipped block, no data are encoded other than an indication to tell that such a block is to be decoded as Skip mode. Hereinafter, we try to discuss the impact of skipped block size.

For an $8 \times 8$ DCT, Sun et al. [9] indicated that if the STD $\left(\sigma_{R}\right)$ of the DCT coefficients $(R)$ and the quantization step $(\Delta)$ satisfy $\sigma_{R}<0.83 \Delta$, the corresponding DCT coefficient would be quantized to zero. It is known that usually the DC coefficient is larger than AC coefficients, and therefore, if the DC coefficient is quantized to zero, such block can be considered as a candidate of a skipped block. In [9], the STD $\left(\sigma_{R-D C}\right)$ of the DC coefficient is given by

$$
\sigma_{R-D C}=\sqrt{\left[A V A^{T}\right]_{0,0} \cdot\left[A V A^{T}\right]_{0,0}}
$$

where $A$ is the DCT transform matrix, $[\cdot]_{0,0}$ denotes the $(0,0)-t h$ component of the matrix, and $V$ is expressed as

$$
V=\sigma_{r}^{2}\left[\begin{array}{ccccc}
1 & \rho & \rho^{2} & & \rho^{7} \\
\rho & 1 & \rho & \ldots & \\
\rho^{2} & \rho & 1 & & \\
& & \vdots & \ddots & \\
\rho^{7} & & & & 1
\end{array}\right]
$$

where $\rho$ is a small constant. Assume that $\rho=0.9$, we can see that if

$$
\sigma_{r}<0.134 \Delta
$$

Such a block would be encoded using Skip mode. For super-HD video sequences, a larger number of blocks satisfy Eq.6, and therefore, we can save more bits by defining $32 \times 32$ P_Skip and $16 \times 16$ B_Skip blocks.

\section{EXPERIMENTAL RESULTS FOR THE EXTENSIONS OF STANDARD H.264/AVC FREXT}

In the previous section, we know of the possibilities of enhanced coding performance of H.264/AVC FRExt with superHD visual content. In this section, we extend the standard H.264/AVC FRExt by using larger block size, search range and skipped block size. The benefits are verified against the standard H.264/ AVC FRExt and Motion JPEG2000 for super-HD video coding. Three $4096 \times 2160$ clips "Pedestrian area", "Crowd Run" and "Park joy" (as shown in Fig. 1) are tested. 


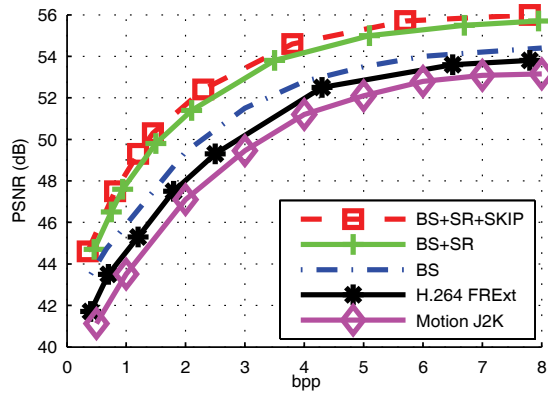

(a) Pedestrian area

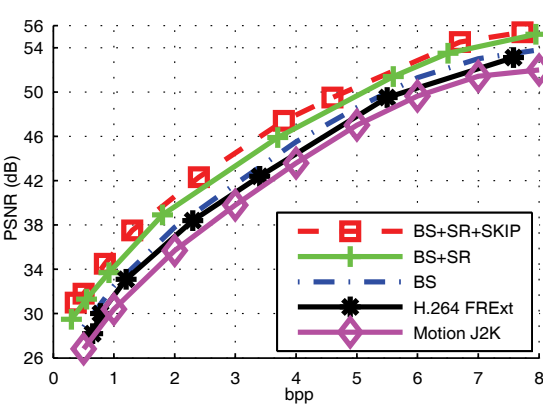

(b) Crowd Run

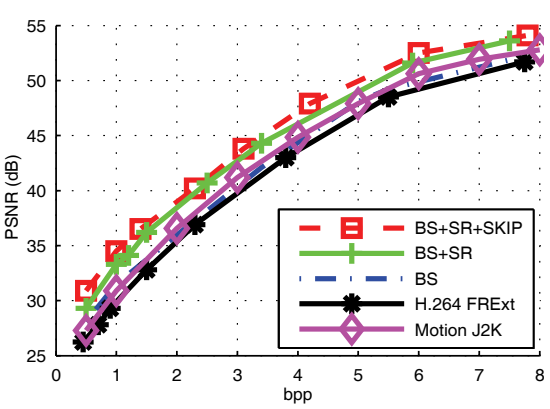

(c) Park joy

Fig. 5: The rate-distortion (R-D) performance (luma component) comparison among the extended H.264/AVC FRExt, standard H.264/AVC FRExt and the Motion JPEG2000.

For the extended H.264/AVC FRExt, JM 16.2 [5] at Level 5.1 has been revised accordingly. Each video frame was partitioned into $32 \times 32$ enlarged macroblocks, which were further partitioned into $32 \times 16,16 \times 32,16 \times 16,16 \times 8,8 \times 16,8 \times 8$, $8 \times 4,4 \times 8$, and $4 \times 4$ blocks; the maximum SR for ME was set to 128 ; the sizes of P_Skip and B_Skip blocks were $32 \times 32$ and $16 \times 16$; Other parameters are set the same as those described in Section 2.

The experiments were conducted as follows: we first extended the standard H.264/AVC FRExt by using enlarged block size (denoted as "BS" in Fig.5); and then the maximum SR was further expanded (denoted as "BS+SR" in Fig.5); finally, larger Skipped block size was configured (denoted as "BS+SR+SKIP" in Fig.5). The comparison results were shown in Fig.5 for the proposed extensions of H.264/AVC FRExt against the standard H.264/AVC FRExt, as well as the Motion JPEG2000. We can see that $2.0 \mathrm{~dB}$ to $3.5 \mathrm{~dB}$ (with an average of $3.0 \mathrm{~dB}$ ) PSNR gain (for "BS+SR+SKIP") has been achieved for the extended H.264/AVC FRExt over standard H.264/AVC FRExt (Motion JPEG2000 has similar average performance with the standard H.264/ AVC FRExt). The experiments also show that an average of $0.7 \mathrm{~dB}, 1.8 \mathrm{~dB}$ and $0.5 \mathrm{~dB}$ PSNR gain has been achieved by enlarged BS, SR and Skipped block, respectively; these are the indication that each of the three aspects contributes actively to the overall performance of the extended H.264/ AVC FRExt scheme.

From Fig.5, it can be concluded that the extensions have significantly improved the coding efficiency of the standard H.264/AVC FRExt. The reasons for the improvement are that: (1) As shown in Fig.3, super-HD sequences are more homogeneous toward the same visual content, and therefore, a larger BS can better exploit the homogeneity and thus reduce the codestream for motion vectors while keeping good performance of motion compensation; (2) For super-HD sequences, motion among neighboring frames is higher with the same visual content, and therefore a larger SR captures the moving objects more accurate and brings about enhancement to the reduction of temporal redundancy; (3) More blocks has been encoded by Skip mode in super-HD sequences, and larger skipped block size can further improve the coding efficiency of the Skipped blocks.

\section{CONCLUSION}

In this paper, the rate-distortion (R-D) performance of the H.264/AVC FRExt and the Motion JPEG2000 has been first compared for super-HD video sequences. For super-HD video coding, the advantage of inter-fame based standard H.264/AVC FRExt is not obvious in comparison with the Motion JPEG2000. We analyzed the reasons for this phenomenon and discussed the impact of macroblock partition, motion search range and skipped block size. Based on the results of theoretical analysis and the insight obtained, standard H.264/AVC FRExt has been extended for superHD video compression. The simulation shows that $2.0 \mathrm{~dB}$ to $3.5 \mathrm{~dB}$ of PSNR gain has been achieved in comparison with the standard H.264/AVC FRExt, as well as the Motion JPEG2000 (since the standard H.264/AVC FRExt and the Motion JPEG2000 perform similarly on average for superHD content.

\section{REFERENCES}

[1] DCI, "Digital Cinema System Specification Version 1.2," Mar. 2008, Available: http://www.dcimovies.com.

[2] M. Smith and J. Villasenor, "Intra-frame JPEG2000 vs. Interframe Compression," SMPTE Conf., pp. 1-9, Oct. 2004.

[3] B.X. Shi and C. Xu, "Comparison between JPEG2000 and H.264 for Digital Cinema," ICME'08, pp.725-728, Jun. 2008.

[4] G. Baruffa and F. Frescura, "Performance Assessment of JPEG2000 based MCTF and H.264 FRExt for Digital Cinema Compression," IEEE Int. Conf. DSP, pp. 11-15, Jul. 2009.

[5] K. Suehring, "H.264/AVC Reference Software - JM 16.2," Available: http://iphome.hhi.de/suehring/tml.

[6] http://en.wikipedia.org/wiki/H.264.

[7] D. Taubman, "Kakadu Reference Software, Version. 6.3.1", Available: http://www.kakadusoftware.com.

[8] N.S Jayant and P. Noll, Digital Coding of Waveforms: Principles and Applications to Speech and Video, Prentice Hall, Englewood Cliffs, NJ, 2007.

[9] M.T. Sun and I.M. Pao, "Statistical Computation of Discrete Cosine Transform in Video Encoders," J. Visual Coтmu. Image Present., 9(2): 163-170, Jun. 1998. 